\title{
Structural characterization of five-component food grade oil-in-water nonionic microemulsions $\dagger$
}

\author{
A. Yaghmur, $\ddagger^{a b}$ L. de Campo, ${ }^{b}$ A. Aserin, ${ }^{a}$ N. Garti ${ }^{a}$ and O. Glatter*b \\ a Casali Institute of Applied Chemistry, The Hebrew University of Jerusalem, Jerusalem \\ 91904, Israel \\ ${ }^{\boldsymbol{b}}$ Institute of Chemistry, Physical Chemistry, University of Graz, Heinrichstrasse 28, \\ A-8010 Graz, Austria. E-mail: otto.glatter@uni-graz.at; Fax: +43 316380 9850; \\ Tel: +433163805433
}

Received 12th November 2003, Accepted 30th January 2004

First published as an Advance Article on the web 27th February 2004

\begin{abstract}
The microstructure of a multi-component oil-in-water $(\mathrm{O} / \mathrm{W})$ microemulsion, to serve as a microreactor or a solubilization vehicle for food applications, has been studied using small angle scattering X-rays (SAXS) and neutron (SANS) techniques. Significant structural changes along selected aqueous dilution lines in the $\mathrm{O} / \mathrm{W}$ microemulsions were determined. We found that the droplets' size is affected by increasing the water content, the oleic phase concentration and content (mixture of $R(+)$-limonene and ethanol), and the nature of the surfactant (Brij 96v and Tween 60). The micellar size increases with increasing the aqueous phase content in both, Brij 96v-based and Tween 60-based systems. Replacing Brij 96v by Tween 60 at constant weight leads to larger microemulsion droplets. The increase in the surfactant concentration of these systems, as expected, leads to smaller interaction radii and to higher values of the number particle density. Increasing ethanol content in the oleic phase $(R(+)$-limonene plus ethanol) decreases the effective volume fraction, and causes reduction in the micellar size at decreasing surfactant aggregation number as a result of its redistribution between the interfacial film and the continuous aqueous phase. The SANS investigations allowed a focus on the main effects of propylene glycol (PG) and ethanol (EtOH). Both hydrophilic molecules have similar tuning properties on the microstructure. Both decrease the droplet size, render them more globular, and increase the number of micelles. It was concluded from these results that both alcohols are partially incorporated into the interface (most of it stays in the aqueous phase). The only observed difference in the investigated samples was that upon replacing the aqueous phase partially with PG, the effective volume fractions of the dispersed phase was decreased, while it remained unchanged when it was partially replaced by EtOH. PG and EtOH seem to influence the microstructure independently.
\end{abstract}

\section{Introduction}

Understanding the phase behavior and microstructure of microemulsions is an important fundamental aspect of the utilization of these structured fluids efficiently in industrial applications. Water-in-oil $(\mathrm{W} / \mathrm{O})$ and oil-in-water $(\mathrm{O} / \mathrm{W})$ microemulsions, formulated with hydrocarbons as oils and ethoxylated alkyl ethers $\left(\mathrm{C}_{i} \mathrm{E}_{j}\right)$ as surfactants, have been the topic of numerous studies. ${ }^{1,2}$ Today, we have a more profound understanding of the phase behavior and microstructure of these systems. ${ }^{2-6}$ However, the microemulsions in industrial applications are rarely simple ternary systems, but more often complicated multicomponent systems. It is not always clear whether those complex systems have similar droplet sizes and shapes and what the roles of the additional components in stabilizing the interface are. Systematic investigations should be useful to understand the microstructure and the role of the different components on the system.

In recent years, few attempts have been made to formulate and characterize microemulsions that can be used for food and pharmaceutical purposes. ${ }^{7,8}$ In this effort, normal alkanes have been replaced by oils acceptable in food (or

$\dagger$ Presented at the 17th Conference of the European Colloid \& Interface Science Society, Firenze, Italy, September 21-26, 2003.

$\ddagger$ Current address: Institute of Chemistry, Physical Chemistry, University of Graz, Austria. pharmaceuticals) industry, and the majority of preparations are oil continuous. These papers focused on studying the ability of formulating a microemulsion with triglycerides ${ }^{9-14}$ and perfumes ${ }^{15-17}$ as the oil component. Joubran and coworkers $^{18,19}$ have studied the phase behavior and microstructure of water-in-triglycerides $(\mathrm{W} / \mathrm{O})$ microemulsions based on polyoxyethylene (40) sorbitanhexaoleate. They found that the monophasic area of these systems was strongly dependent on temperature and aqueous phase content. Lawrence and coworkers ${ }^{11,12}$ examined the solubilization of a range of triglycerides and ethyl esters in an oil-in-water microemulsion system with nonionic surfactants. They concluded that the solubilization capacity depends not only on the nature of the surfactants but also on the nature of the oil.

There are very few surfactants that can be used in food formulations. ${ }^{7}$ In this respect, Tweens, (ethoxylated derivatives of sorbitan esters), are an interesting family of surfactants. The substitution of the hydroxyl groups on the sorbitan ring with bulky polyoxyethylene groups increases the hydrophilicity of the surfactant. The ability of Tweens to form microemulsions for pharmaceutical applications has been studied by few authors. ${ }^{20-24}$ An increased solubility of lipophilic drugs in the microemulsion regime was observed and explained by the penetration of these drugs into the interfacial film. ${ }^{22-24}$

Our recent works ${ }^{25,26}$ have specifically dealt with the improved water and oil solubilization in the presence of polyols (propylene glycol, PG, and glycerol, Gly) and short-chain 
alcohol (ethanol, EtOH) in U-type nonionic $\mathrm{W} / \mathrm{O}$ and $\mathrm{O} / \mathrm{W}$ food (and cosmetic) microemulsion systems. We found that alcohols and polyols destabilize the liquid crystalline phase and extend the isotropic region to higher surfactant concentrations. Of interest is the ability of these additives to provide a large monophasic system in which high amounts of oil and water can be solubilized. The pseudo-ternary phase diagrams for $R(+)$-limonene-ethanol-water-PG systems based on Tween 60 (food-grade system) and Brij $96 \mathrm{v}\left(\mathrm{C}_{18: 1}(\mathrm{EO})_{10}\right.$, this system is acceptable in cosmetics) are shown in Fig. $1 .^{25,26}$ In these systems which offer great potential in practical formulations, it is possible to follow the structural evolution of the microemulsion system from aqueous phase-poor to aqueous phase-rich without encountering phase separation. The fivecomponent systems have been utilized as selective microreactors for the generation of sulfur-containing aroma compounds. ${ }^{27}$ Recently, these complex systems have attracted a great deal of attention because of their potential application as reservoirs for enhanced solubilization of active hydrophobic or hydrophilic food ingredients. ${ }^{28,29}$ We recently examined, by a sub-zero DSC technique the nature of the water and its thermal behavior in these systems, ${ }^{30}$ and their microstructure by NMR pulsed-gradient-spin-echo (PGSE) nuclear magnetic resonance (NMR), conductivity, and rheology. ${ }^{31}$ In those previous works, we found that the microemulsion transforms from a water-in-oil $(\mathrm{W} / \mathrm{O})$ microemulsion, to a bicontinuous phase and to an oil-in-water $(\mathrm{O} / \mathrm{W})$ microemulsion. ${ }^{30,31} \mathrm{We}$ reported that the surfactant/alcohol/PG can strongly bind water in the inner phase so that it freezes below $-10^{\circ} \mathrm{C}$ and acts in part as "bound" water and in part as "non-freezable" water. ${ }^{30}$ Even after complete inversion to $\mathrm{O} / \mathrm{W}$ microemulsions the water in the continuous phase is strongly interacting with the PG/surfactant and remains bound or non-freezable.

(a)

$$
\text { R(+)-limonene / EtOH (1/1) }
$$

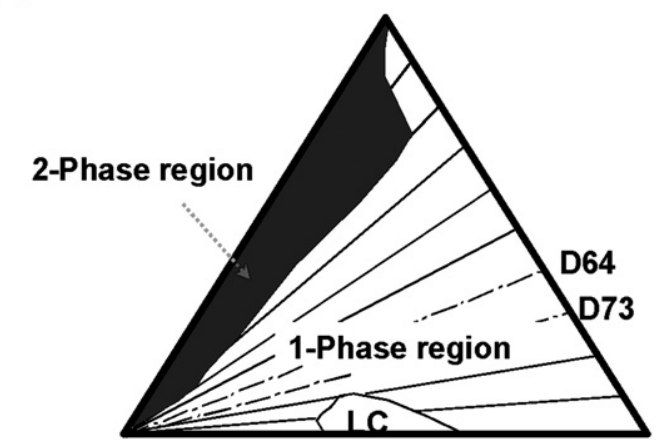

Water / PG (1/1)

Brij 96v

(b)

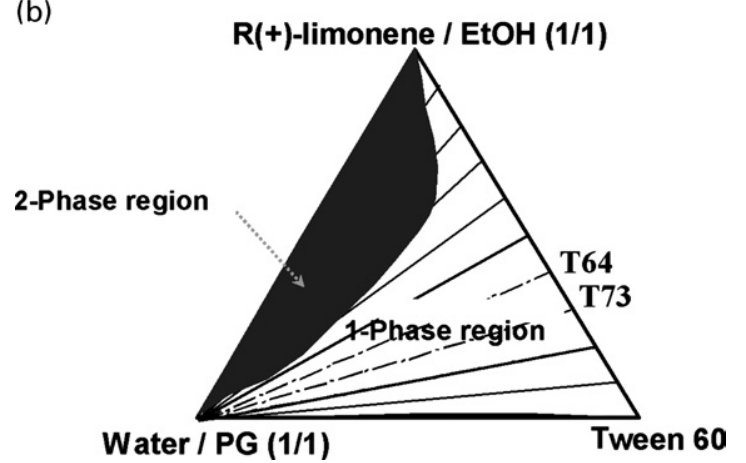

Fig. 1 Phase diagrams of the system: water $/ \mathrm{PG} / R(+)$-limonene/ $\mathrm{EtOH} /$ ethoxylated nonionic surfactant at $25^{\circ} \mathrm{C}$ with constant weight ratio of water/PG $(1 / 1)$ and a constant weight ratio of $R(+)$ limonene/EtOH (1/1). Ethoxylated surfactants used in these phase diagrams were: (a) Brij 96v; (b) Tween 60.
It was found also that the ratio of the water self-diffusion coefficient, $\mathrm{D}_{\mathrm{W}}$, to the PG self-diffusion coefficient, $\left(\mathrm{D}_{\mathrm{PG}}\right)$ (termed the L-ratio) was sensitive to the aqueous phase content. In the $\mathrm{O} / \mathrm{W}$ microemulsions, the water/PG mixture diffuses as hydrogen bonded entity (molecular diffusion controlled), while in the $\mathrm{W} / \mathrm{O}$ microemulsions, the $\mathrm{PG}$ partitions, in part, at the interface and the dominant diffusion process is neither aggregate nor molecular controlled. ${ }^{31}$

Studies on the effect of water-soluble polyols (e.g., propylene glycol, glycerol) and their binary mixtures on the phase behavior and microstructure of low-molecular weight surfactants ${ }^{32-36}$ (such as polyoxyethylene-type nonionic surfactants) and amphiphilic copolymers have been recently reported..$^{37,38}$ Replacement of water by polar solvents in ionic and nonionic systems leads to an increase in critical micellization concentrations ${ }^{39-41}(\mathrm{cmc})$, a reduced tendency for structure formation, ${ }^{42}$ and a decrease in stability of liquid crystalline phases. ${ }^{32,33}$ These effects have been interpreted in terms of changed water solvent quality. It was reported that the addition of these solvents could lead to dehydration (hydration) of the poly(ethylene oxide) chain of the surfactants and corresponding decrease (increase) of the surfactant hydrophilicity. ${ }^{33,37,38}$ The phase behavior and structure of nonaqueous microemulsions have been investigated. ${ }^{34-36,43}$ The phase behavior of these systems was found similar to those of water microemulsions. ${ }^{34-36}$

In this context, the present work has fundamental significance toward an improved understanding of the effects of water-soluble polyols and short-chain alcohols on the microstructure of five-component nonionic microemulsions and has also practical significance, e.g., in food formulations. This investigation aims to produce a system where the type of microstructure can be modulated by varying the aqueous and/or the oleic phase composition and content. In the present study, we extend our knowledge on the structure of our fivecomponent microemulsion systems by investigating them by using small angle scattering of X-rays (SAXS) and neutrons (SANS). These techniques are very powerful tools for the characterization of microemulsions and have been useful in the structural characterization of different ionic and nonionic microemulsions. ${ }^{3-5,44}$ For the evaluation of the scattering data from these dense systems, we use the recently developed generalized indirect fourier transformation (GIFT) method. ${ }^{45-48}$

\section{Materials and methods \\ Materials}

Brij 96v (polyoxyethylene (10) oleyl alcohol, $\left(\mathrm{C}_{18: 1} \mathrm{E}_{10}\right)$ ) was purchased from ICI, Specialty Chemicals (Essen, Germany). Ethoxylated sorbitan esters in use were Tween 60 (ethoxylated-20 EO-sorbitan monostearate), and Tween 80 (ethoxylated-20 EO-sorbitan monooleate). These Tweens as well as $R(+)$-limonene $(98 \%)$ were of commercial grade and purchased from Sigma Chemical Co. (St. Louis, Missouri, USA). Ethanol $(\mathrm{EtOH})$ was obtained from Frutarom (Haifa, Israel). Propylene glycol, PG (1,2-propanediol, BDH, Poole, England) was used. These materials were used without further purification. The water used was double distilled.

For SANS measurements: the deuterated propylene glycol$\mathrm{d}_{8}(99 \%)$ (in the following denoted as d-PG) and the deuterated ethanol-d $\mathrm{d}_{6}(99 \%)$ (denoted as d-EtOH) were obtained from Cambridge Isotope Laboratories (Andover, Massachusetts, USA), $\mathrm{D}_{2} \mathrm{O}$ was supplied by Aldrich (100\%).

\section{Phase diagrams}

The five-component systems were described on pseudo-ternary phase diagrams. They were constructed as reported recently ${ }^{25}$ at the temperature of $25^{\circ} \mathrm{C}$. The accuracy in the location of the phase boundaries is within $4 \mathrm{wt} . \%$. The aqueous phase 
dilution lines that have been investigated in this study are shown in Fig. 1.

\section{Scattering techniques}

A. Small-angle neutron scattering measurements. The small-angle neutron scattering (SANS) experiments were carried out using the SANS-I instrument at the SINQ neutron spallation source in Paul Scherrer Institute (PSI), Switzerland. The ranges of $0.06 \mathrm{~nm}^{-1}<q<2.45 \mathrm{~nm}^{-1}$ were covered for the scattering vectors by two sample-to-detector distances $(d=2$ and $18 \mathrm{~m}$ ). The neutron mean wavelength was $0.8 \mathrm{~nm}$ at PSI. The wavelength resolution was $10 \%$ (full width at half maximum value). The samples were kept in stoppered quartz cells (Hellma, Germany) with a path length of $1 \mathrm{~mm}$. The raw spectra were corrected for background from the solvent, sample cell, and electronic noise by conventional procedures. The two-dimensional scattering spectra were azimuthally averaged and corrected for detector efficiency, dividing them by the incoherent scattering spectrum of pure water. Data were put on absolute scale using water as a secondary standard.

B. Small-angle X-ray scattering (SAXS) measurements. The SAXS set-up consists of a modified Kratky compact camera (Anton-Paar, Graz, Austria) with slit collimation and transparent beam stop in combination with a Siemens M18X rotating anode operating at $40 \mathrm{kV}$ and $100 \mathrm{~mA}$ (Bruker AXS, Karlsruhe, Germany). A Göbel mirror is used to convert the divergent polychromatic X-ray beam into a focused beam of $\mathrm{Cu} \mathrm{K} \mathrm{K}_{\alpha}$ radiation $(\lambda=0.154 \mathrm{~nm})$. The $2 \mathrm{D}$ scattering pattern is recorded by an imaging-plate detector (model Fuji BAS1800 from Raytest, Straubenhardt, Germany) and integrated to the one-dimensional scattering function $I(q)$ using AIDA software. A detailed description of the experimental set-up is given in a previous paper. ${ }^{49}$ The geometric effects of the line shaped primary beam (slit smearing) is taken account of during evaluation with GIFT. ${ }^{50}$

Small angle scattering (SAS) was used to obtain information on the size and shape of the micellar aggregates and their internal structure. SAS experiments use the interference effect of wavelets scattered from different materials in a sample (different scattering length densities). The result is an angle dependent scattering curve $I(q)$, in which $q$ is the length of the scattering vector, defined by $q=(4 \pi / \lambda) \sin \theta / 2, \lambda$ being the wavelength and $\theta$ the scattering angle.

The scattering curve $I(q)$ of highly diluted samples is just the form factor of the droplets and can be interpreted in real space in terms of their pair distance distribution functions (PDDF). ${ }^{51,52}$ The PDDF or $p(r)$ is the Fourier transform of the form factor. A quantitative numerical analysis of properly pretreated data, involving Fourier transformation, provides information not available elsewhere. The PDDF represents a histogram of distances inside the particle, weighted with the scattering length density differences, and it approaches zero at the maximum particle dimension. For globular micelles with constant contrast, the PDDF is a bell-shaped function with its maximum at about half of the diameter. The area under the PDDF is proportional to the forward-scattering intensity and thus to the squared scattering length density difference, i.e., "contrast", between the particles and the solvent and to the concentration of the dispersed phase (number density).

All measured samples are in a concentration range where the conventional evaluation techniques cannot be used. Due to the high volume fractions involved, the interaction between the particles in solution must also be taken into account.

In the case of interacting globular particles, the scattering intensity $I(q)$ can be written as the product of the particle form factor $P(q)$ and the structure factor $S(q) .{ }^{53} S(q)$ describes the interparticle interference. For ideally diluted solutions, $S(q)$ is a constant and equals one. The scattering curves show the development of a structure factor with a pronounced maximum for increasing concentrations, superimposed on the particle form factor. The evaluation of such concentrated solutions is now possible with the recently developed GIFT method. ${ }^{45,50,54,55}$ With this technique it is possible to determine simultaneously the form factor and the structure factor. This method is model free in respect to the form factor and is based on a hard sphere model for the structure factor and, strictly speaking, it is only valid for spherical and monodisperse particles. However, the effects of polydispersity can be approximated. Recent applications have shown that the GIFT method can be used far beyond the theoretical limits, ${ }^{47,48,54}$ so the technique can be used also for inhomogeneous, nonglobular and polydisperse systems. For instance, it was possible to follow sphere to rod transition of binary and ternary systems. ${ }^{55}$ The factorization of $I(q)$ into $P(q)$ and $S(q)$ works well for volume fractions up to $50 \%$ for monomodal distribution of spheres. ${ }^{56}$ However, it may break down for high volume fractions of anisotropic and highly polydisperse systems. The common feature of all these applications is the fact that the particle form factor can be determined without any model while a rather simple model is used to describe particle interactions represented by the structure factor. The typical parameters for the structure factor model for uncharged systems are the mean interaction radius $\left(R_{\mathrm{HS}}\right)$, the effective volume fraction of the dispersed phase $\left(\phi_{\mathrm{eff}}\right)$, and a polydispersity parameter $(\mu)$. The polydispersity index, $\mu$, is defined as the ratio between the width of a Gaussian distribution and its maximum value $\left(R_{\mathrm{HS}}\right){ }^{50}$ The most important parameter determined in these calculations is $R_{\mathrm{HS}}$. It is half the center-to-center distance between two micelles at closest contact. In the hard sphere model used, polydispersity is approximated by averaging structure factors for a size distribution of different $R_{\mathrm{HS}}$-values at constant volume fraction $\left(S_{\text {ave }}\right)$. This approximation has no impact on the forward scattering of the structure factor, but only on the width of the interaction peak. It has been proven that this model works well when applied to microemulsion data. ${ }^{48} \mathrm{~A}$ comparison with the theoretically expected effective structure factor $S_{\text {eff }}$ has been published recently. ${ }^{54}$ It should be noted that by applying this averaged hard sphere structure factor $\left(S_{\text {ave }}\right)$ to our complex microemulsion data, we do not expect to receive accurate absolute values on the volume and interaction radius, (especially as soon as the droplets are elliptical or slightly polydisperse); however, the parameters give us the possibility to follow trends in the distinct sample lines. The errors in the relative values of $\mathrm{R}_{\mathrm{HS}}$ in all samples of the present study are in the range of $2 \%$, and for $\phi_{\text {eff }} \geqslant 0.2$ approximately $3 \%$. The error in the values of $\phi_{\text {eff }}$ is higher, when the structure factor is less pronounced $\left(\phi_{\text {eff }}<0.2\right)$. The polydispersity parameter, $\mu$, was found to be very low for all samples and does not have much impact in this range.

\section{Results and discussion}

\section{Small angle X-ray scattering (SAXS)}

Microemulsions based on Brij 96v and Tween 60, respectively, were investigated along various aqueous phase dilution lines that are shown in Fig. 1 as dashed-dotted lines. This enables us to compare the scattering pattern of different microemulsion samples in dependence of the surfactant (Brij 96v vs. Tween $60)$, the aqueous phase content and the ratio of surfactant to oleic phase. In some samples also the impact of oleic phase composition $(R(+)$-limonene plus ethanol) was investigated.

It should be noted that, in this study, many of the Tween 60based microemulsions have intrinsic difficulties, when investigated by SAXS: the overall contrast of these systems is quite often close to zero which leads to a very low forward scattering of the form factor. At the same time, the forward scattering of 
the structure factor for such dense systems is also very low, which renders a separation of these two factors very difficult or impossible. The obtained structure factors for Tween 60 based systems turned out to be stable during evaluation (i.e., independent from variation in the program input parameters) and could be used to gain information on the system, while the form factors could not be interpreted in an unambiguous way. To achieve a correct interpretation of those, it will be necessary to perform further SANS experiments as supplement, which is part of our planned future activities. In the present work, therefore, we focus mainly on the evaluation and interpretation of the scattering data from Brij 96v-based systems.

A. Effect of aqueous phase (water plus PG) content in two microemulsion systems. A series of scattering curves of Brij 96v and Tween 60 systems along the dilution lines D64 and T64 are presented in Fig. 2. In the present study, the experimental scattering data are typically shown as raw data after the subtraction of the blank scattering without further manipulations like de-smearing of the instrumental broadening. We found that the fit of the GIFT evaluation procedure is very good over the whole measured $q$-range, including very high and low q regimes as shown in the representative example in Fig. 3a. Typically the fit is clearly within the size of the symbols; therefore the fit lines have not been shown in all other figures. These dilution lines contain a constant weight ratio of $R(+)$-limonene-ethanol (EtOH)-surfactant (1:1:3) and are characterized by single continuous microemulsion regions (transition from oil-rich to aqueous phase-rich microemulsions without the occurrence of phase separation). In this unique Winsor IV isotropic region, the system transforms from a $\mathrm{W} / \mathrm{O}$ microemulsion via a bicontinuous phase to an $\mathrm{O} / \mathrm{W}$ microemulsion. ${ }^{30,31}$ In both microemulsion systems (based on Brij 96v and Tween 60), the same trend in the scattering
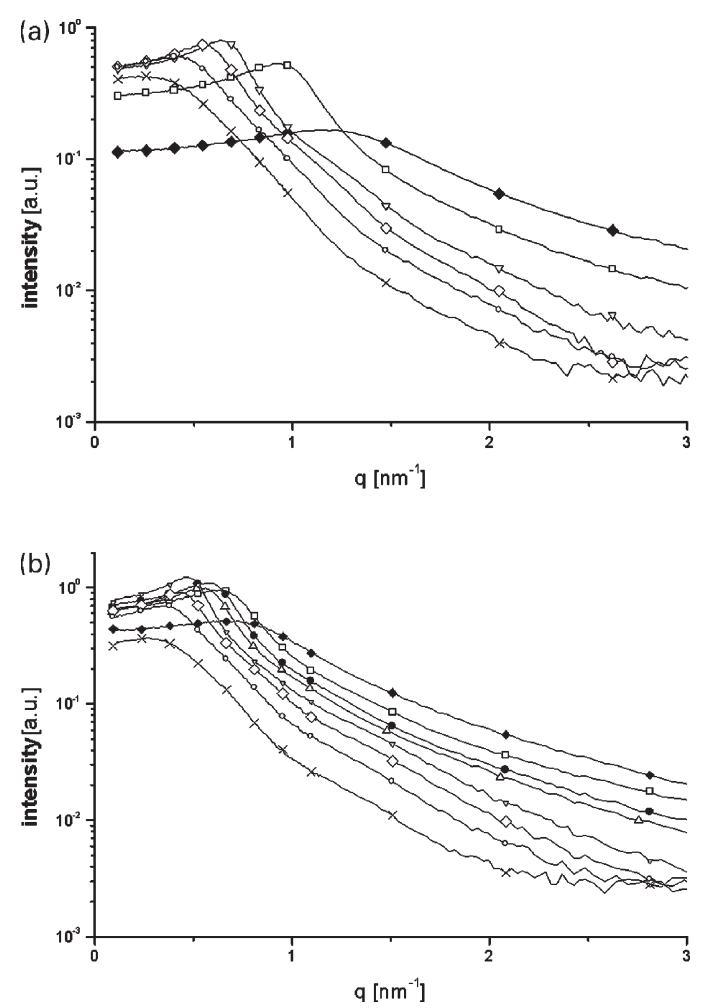

Fig. 2 (a) Experimental slit smeared small-angle X-ray scattering curves after subtraction of the background and solvent scattering for the samples alone the dilution lines T64 (a), and D64 (b), respectively. The aqueous phase (water plus PG at constant weight ratio of $1 / 1$ ) content of the investigated samples: $(\diamond) 10 \mathrm{wt} . \%,(\square) 30 \mathrm{wt} . \%,(\bullet)$ 40 wt. $\%,(\triangle) 50$ wt. $\%,(\nabla) 60$ wt. $\%,(\diamond) 70$ wt. $\%,(\bigcirc) 80$ wt. $\%$, and (×) 90 wt. $\%$.
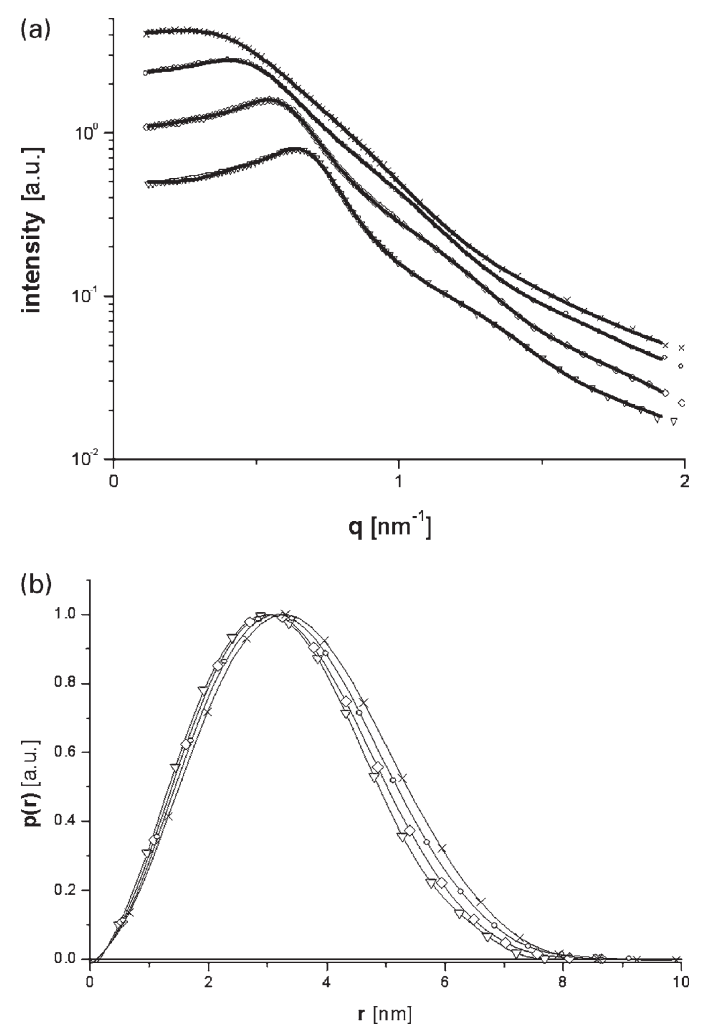

Fig. 3 (a) Experimental slit smeared scattering curves (symbols) for the microemulsion systems in the aqueous-rich region along the dilution line D64, together with the fit (solid line). The aqueous phase (water plus PG at constant weight ratio of $1 / 1$ ) content of the investigated samples: $(\nabla) 60$ wt. $\%,(\diamond) 70$ wt. $\%,(\bigcirc) 80$ wt. $\%$, and $(\times) 90$ wt. $\%$. For better visibility the scattering curves were multiplied by $10^{0}, 10^{1 / 3}, 10^{2 / 3}$ and 10 , repectively, (b) Pair distance distribution functions (PDDFs) for these samples (normalized to the same height)

pattern was observed, as shown in Figs. 2a and 2b. These figures show that with increasing the aqueous phase content (water/PG at a constant weight ratio of $1 / 1$ ), the position of the maximum shifts to lower angles, corresponding to growing distances. Furthermore, the peak initially sharpens and becomes more pronounced to the middle of the line and then broadens again.

It was found for all investigated samples that the maximum of their scattering curves is followed by a Porod tail at high $q$. Porod-like behavior was verified by fitting a linear function to X-ray plots of $I(q) q^{3}$ vs. $q^{3}$, since slit smearing increases the known power-law exponent of $q^{-4}$ by one due to integration over $q$.

The interpretation of these spectra becomes, however, increasingly difficult for higher concentrations of the dispersed phase. In semi-dilute systems with volume fractions above $1 \%$, it is important to take particle interactions into account (using GIFT method ${ }^{45-47}$ ) when evaluating the scattering data. The measured scattering curve can be split into the form factor $(P(q))$ and structure $(S(q))$ factor. It is easier to discuss the form factor in real space, in terms of the pair distance distribution function (PDDF), which is the corresponding Fourier transform of the form factor. The PDDFs (normalized to the same height at the peak maximum) for these microemulsion systems are shown in Fig. 3b. In principle, there is a minor increase in the overall size of all microemulsion droplets with increasing the aqueous phase content. All the swollen micelles have approximately the same shape. Their $p(r)$ functions exhibit the typical shape for slightly polydisperse globular, almost spherical droplets. This can be verified from the PDDF functions, as there is no significant change in the ratio between 
the position of the maximum in PDDFs and the respective maximum dimension $\left(D_{\max }\right)$.

In the Tween 60-based system, along the dilution line T64, good evaluation results of the scattering curves could only be achieved for samples containing more than $80 \mathrm{wt} . \%$ aqueous phase (PDDFs for two of these microemulsions will be discussed in section $\mathrm{C}$ ). The parameters of the structure factors for $\mathrm{O} / \mathrm{W}$ microemulsions based on Brij 96v and Tween 60 (see the dilution lines D64 and T64 in Fig. 1), are presented in Table 1 and Fig. 4. These values must be understood as apparent parameters. In all the investigated samples in this study that are presented in Tables 1 and 2, the polydispersity parameter, $\mu$, is very low (below 7\%); in this regime fluctuations are not significant on absolute scale. As shown in the table, the interaction radius $\left(R_{\mathrm{HS}}\right)$ increases, whereas the effective volume fraction $\left(\phi_{\text {eff }}\right)$ decreases with increasing content of the aqueous phase. It should be noted from Fig. 4a that there is a significant difference in the effective interaction radii $\left(R_{\mathrm{HS}}\right)$ of Brij 96v based systems to those of Tween 60 based systems, while there is no significant difference in the effective volume fractions for both systems. The values of $\mathrm{R}_{\mathrm{HS}}$ are much smaller for the Brij 96v samples. This can be explained as follows: (a) Brij 96v molecules are much smaller than those of Tween 60 (10 EO-units vs. $20 \mathrm{EO}$ units), and (b) replacing the same weight fraction of Brij $96 \mathrm{v}$ by Tween 60 molecules, the number of surfactant molecules is much smaller (which renders less available interface)

It was found that the effective volume fractions $\left(\phi_{\text {eff }}\right)$ derived from the structure factors are very close to the weight fraction of dispersed phase (surfactant + oleic phase), despite the fact that they should be higher since they include the solvation shell of the droplets. So this is just a coincidence, because $\mathrm{EtOH}$ is only formally part of the oil phase, but actually most of it moves to the aqueous phase and part of it penetrates to the interface, which will be discussed later on in greater detail. The growth with dilution can be explained by the fact that the increase in the aqueous phase content leads to a decrease in interfacial concentration of ethanol (redistribution).

In all measured samples, the effective interaction radii derived from interaction properties (structure factors) are in good agreement with the information obtained from the form

Table 1 The structural parameters (effective volume fraction $\phi_{\text {eff }}$, interaction radius $R_{\mathrm{HS}} / \mathrm{nm}$, and polydispersity index $\left.\mu(\%)\right)$ for the averaged Percus-Yevick structure factor $\left(S^{\text {ave }}(q)\right)$ of $\mathrm{O} / \mathrm{W}$ microemulsions along the dilution lines D64, T64, D73, and T73 aqueous phase-rich regime of the phase diagram presented in Fig. 1, and the structure parameters of two $\mathrm{O} / \mathrm{W}$ microemulsions along dilution line E64 in the aqueous phase-rich regime

\begin{tabular}{lllll}
\hline $\begin{array}{l}\text { Aqueous phase } \\
\text { content (wt.\%) }\end{array}$ & & & & \\
& & $\phi_{\mathrm{eff}}$ & $R_{\mathrm{HS}} / \mathrm{nm}$ & $\mu(\%)$ \\
\hline Brij 96v (D64) & 60 & 0.363 & 4.46 & 1.6 \\
& 70 & 0.308 & 4.89 & 1.4 \\
& 80 & 0.205 & 5.40 & 0.5 \\
& 85 & 0.157 & 5.60 & 0.5 \\
& 90 & 0.114 & 5.91 & 6.1 \\
Tween 60 (T64) & 95 & 0.019 & 5.81 & 0.5 \\
& 80 & 0.201 & 6.34 & 0.5 \\
Brij 96v (D73) & 70 & 0.119 & 7.02 & 3.0 \\
& 80 & 0.285 & 4.61 & 1.0 \\
Tween 60 (T73) & 85 & 0.229 & 4.86 & 3.0 \\
& 60 & 0.167 & 5.04 & 3.0 \\
& 70 & 0.294 & 5.158 & 2.6 \\
& 80 & 0.212 & 5.764 & 0.5 \\
Tween 60 (E64) & 95 & 0.030 & 6.130 & 0.5 \\
& 90 & 0.189 & 5.729 & 0.7 \\
& 90 & 0.084 & 6.183 & 0.5 \\
& & & & 6.7 \\
\hline
\end{tabular}
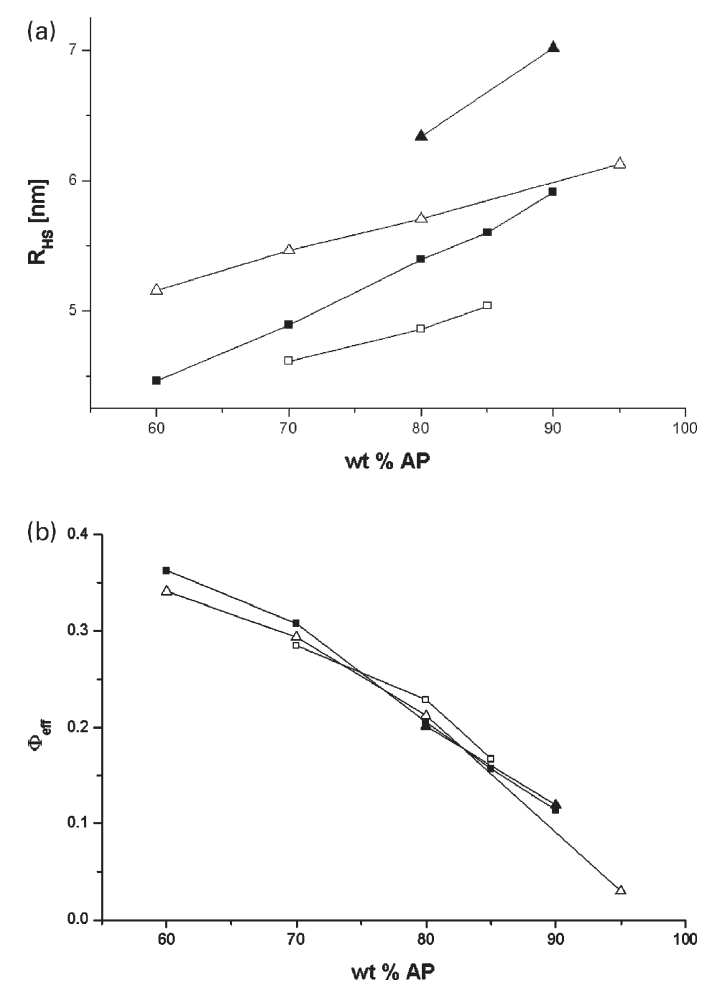

Fig. 4 (a) Effect of aqueous phase content along the Brij 96v-based dilution lines D64 (ם), D73 ( $\square$ ), and the Tween 60-based dilution lines T64 $(\Delta)$, and T73 $(\triangle)$ in the aqueous-rich region on the structure factor parameters as were calculated from the evaluation of SAXS data by GIFT: (a) the interaction radius $\left(R_{\mathrm{HS}}\right)$, and (b) the effective volume fraction $\left(\phi_{\mathrm{eff}}\right)$.

factor (PDDFs): they exhibit the same trend and are slightly larger than half the maximum dimensions from the PDDFs. This can be attributed to the fact that the scattering contrast between the outer shell of the droplets (hydrated PEO-chains) and the surrounding continuous phase is very low. Therefore, we can hardly see this outer shell for the Brij 96v-based systems in the PDDFs from SAXS data.

In the present project, also the scattering data of samples in the lower aqueous phase regime were evaluated by GIFT, and "good estimates" for the size of the occurring structures was found. However, it was decided not to discuss this region at all, because we know that we are encountering bicontinuous phases in the middle of the dilution line, and therefore any kind of hard sphere model is not justified any longer. It was our main idea to focus on $\mathrm{O} / \mathrm{W}$ microemulsions, because these systems were utilized as micro-reactors for the generation of flavors and solubilization of food additives. ${ }^{27-29}$

B. Effect of surfactant concentration and oil phase content. The scattering curves of the samples along the dilution line D73 in the aqueous phase-rich regime are presented in Fig. 5a. While the $p(r)$ functions for dilution line D73 remain unchanged upon dilution (same size and globular shape Fig. $5 \mathrm{~b}$ ), the parameters of the structure factors (also for line T73, Table 1 and Fig. 4) show a very similar trend as D64 and T64 (Table 1). Upon increasing the aqueous phase content along the dilution line D73 and T73, the effective volume fraction decreases, and the microemulsion droplets particles swell $\left(R_{\mathrm{HS}}\right)$ although less pronounced than in line D64 and T64. This swelling cannot be observed in the PDDFs (Fig. 5b), because it is not very pronounced and obviously mostly concerns the (for SAXS) invisible shell of the droplets (see section above). Comparing the PDDFs of line D64 and D73, (Fig. 3b and Fig. 5b) it is obvious that with increasing the surfactant ratio the droplets become smaller, as expected. 
Table 2 The structural parameters (effective volume fraction $\phi_{\mathrm{eff}}$, interaction radius $R_{\mathrm{Hs}} / \mathrm{nm}$, and polydispersity $\mu(\%)$ ) for the averaged Percus-Yevick structure factor $\left(S^{\text {ave }}(q)\right)$ and the number of particles $\left[N_{\mathrm{p}} \mu \mathrm{m}^{-3}\right]$ for the SANS data of the investigated samples by SANS

\begin{tabular}{|c|c|c|c|c|c|c|c|c|c|c|c|c|}
\hline \multirow[b]{2}{*}{ Samples } & \multicolumn{7}{|c|}{ Composition of the investigated samples by SANS } & \multicolumn{4}{|c|}{$\begin{array}{l}\text { Structure parameters of the } \\
\text { investigated samples }\end{array}$} & \multirow[b]{2}{*}{ Comment } \\
\hline & 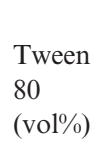 & $\begin{array}{l}\mathrm{AP}= \\
\mathrm{D}_{2} \mathrm{O}+ \\
(\mathrm{d}-) \mathrm{PG} \\
\left(\mathrm{vol}^{\circ} \%\right)\end{array}$ & $\begin{array}{l}R(+)- \\
\text { limonene } \\
\left(\mathrm{vol}^{\circ} \%\right)\end{array}$ & $\begin{array}{l}\text { d-EtOH } \\
\left(\mathrm{vol}^{2} \%\right)\end{array}$ & $\begin{array}{l}\mathrm{D}_{2} \mathrm{O} \\
\left(\mathrm{vol}^{\%} \%\right)\end{array}$ & $\begin{array}{l}(\mathrm{d}-) \mathrm{PG} \\
\left(\mathrm{vol}^{\mathrm{O}} \mathrm{)}\right)\end{array}$ & $\begin{array}{l}\text { PG in } \\
\text { AP } \\
(w t . \%)\end{array}$ & $\Phi_{\text {eff }}$ & $\begin{array}{l}R_{\mathrm{HS}} / \\
\mathrm{nm}\end{array}$ & $\begin{array}{l}\mu \\
(\%)\end{array}$ & $\begin{array}{l}N_{\mathrm{p}} \\
\mu \mathrm{m}^{-3}\end{array}$ & \\
\hline a1 & 19.8 & 80.2 & - & - & 80.2 & - & - & 0.309 & 4.76 & 0.5 & $684 \times 10^{3}$ & \multirow{3}{*}{$\begin{array}{l}\text { Impact of } \\
\text { PG on } \\
\text { aqueous } \\
\text { micelles } \\
\text { based on } \\
\text { Tween } 80\end{array}$} \\
\hline $\mathrm{a} 2$ & 19.8 & 80.2 & - & - & 60.7 & $19.5^{a}$ & 24.3 & 0.291 & 4.45 & 0.4 & $789 \times 10^{3}$ & \\
\hline a3 & 19.8 & 80.2 & - & - & 40.8 & $39.4^{a}$ & 49.1 & 0.279 & 4.19 & 1.0 & $904 \times 10^{3}$ & \\
\hline $\mathrm{a} 4$ & 19.8 & 79.2 & 1.0 & - & 60.0 & 19.2 & 24.3 & 0.295 & 4.83 & 0.4 & $625 \times 10^{3}$ & \multirow{3}{*}{$\begin{array}{l}\text { Impact of } \\
\text { EtOH } \\
\text { on Tween } \\
\text { 80-based } \\
\text { micro- } \\
\text { emulsions }\end{array}$} \\
\hline a5 & 19.8 & 74.2 & 1.0 & 5.0 & 56.2 & 18.0 & 24.3 & 0.297 & 4.67 & 1.0 & $697 \times 10^{3}$ & \\
\hline a6 & 19.8 & 69.2 & 1.0 & 10.0 & 52.4 & 16.8 & 24.3 & 0.290 & 4.52 & 0.4 & $751 \times 10^{3}$ & \\
\hline a7 & 19.80 & 79.20 & 1.0 & - & 79.20 & - & - & 0.320 & 5.09 & 3.7 & $579 \times 10^{3}$ & \multirow{3}{*}{$\begin{array}{l}\text { Impact of } \\
\text { EtOH } \\
\text { on Tween } \\
\text { 80-based } \\
\text { micro- } \\
\text { emulsions } \\
\text { free from } \mathrm{PG}\end{array}$} \\
\hline a8 & 19.80 & 74.20 & 1.0 & 5.0 & 74.20 & - & - & 0.326 & 4.97 & 4.0 & $634 \times 10^{3}$ & \\
\hline a9 & 19.80 & 69.20 & 1.0 & 10.0 & 69.20 & - & - & 0.324 & 4.81 & 4.4 & $695 \times 10^{3}$ & \\
\hline
\end{tabular}
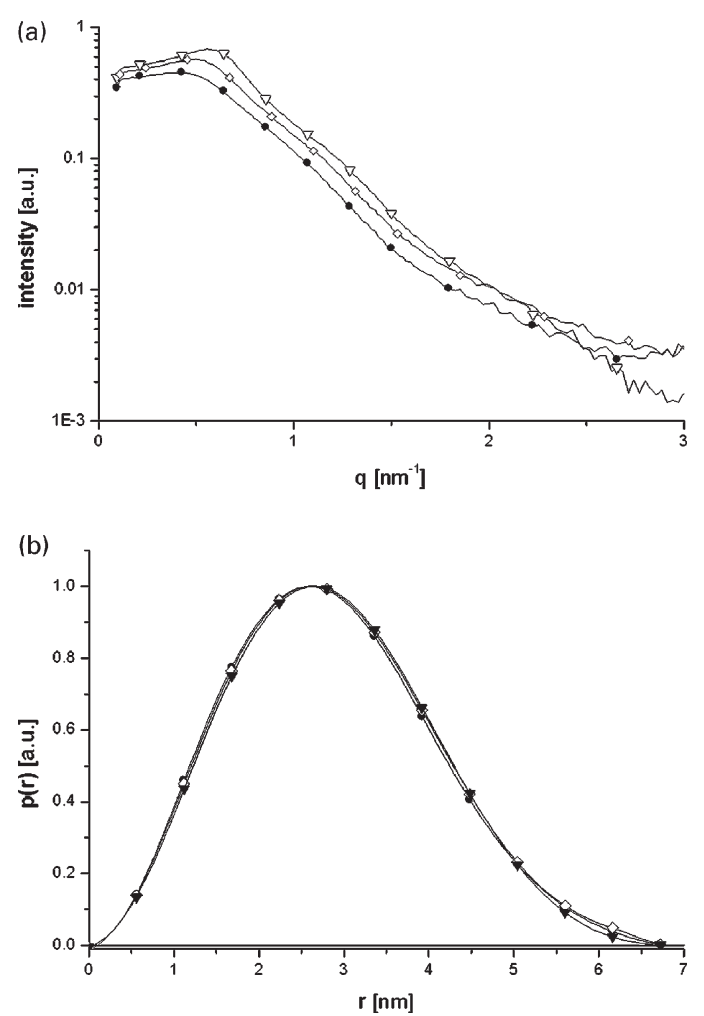

Fig. 5 (a) Experimental slit smeared scattering curves after subtraction of the background and solvent scattering for the samples along the dilution lines D73. (b) The pair distance distribution functions (PDDFs) for the microemulsion systems in the aqueous-rich region along the same dilution line. The aqueous phase (water plus PG at constant weight ratio of $1 / 1$ ) content of the investigated samples: $(\nabla) 70$ wt. $\%,(\diamond) 80$ wt. $\%$, and $(\bullet) 85$ wt. $\%$.
Figs. $4 \mathrm{a}$ and $4 \mathrm{~b}$ summarize the effect of surfactant and oil concentration on the interaction properties derived from the structure factor: the effective interaction radii $\left(R_{\mathrm{HS}}\right)$ and effective volume fractions $\left(\phi_{\text {eff }}\right)$ in $\mathrm{O} / \mathrm{W}$ microemulsion systems based on Brij 96v and Tween 60. The surfactant/oleic phase weight ratio is $6 / 4$ (for dilution lines D64 and T64), and 7/3 (for dilution lines D73 and T73). With increasing surfactant concentration (moving from dilution line D64 to D73 and from T64 to T73), the effective volume fractions approximately stay constant irrespective of the mixing ratio. At the same time $R_{\mathrm{HS}}$ is strongly decreased with increasing surfactant content as expected (in agreement with smaller sizes indicated by the PDDFs), as the later generates more available interfacial area. Again, all microemulsion droplets based on Tween-60 are larger than those based on Brij 96v.

Focusing on the effect of dilution with aqueous phase on the interaction properties, it was observed that in all dilution lines there is almost the same decrease in volume fraction (Fig. 4b) but the linear slight increase of the interaction radii (Fig. 4a) is more pronounced for the dilution lines D64 and T64 (full symbols) than in D73 and T73 (empty symbols). We were not able observe this apparent growth in the PDDFs of the lines D73 (Fig. 5b) and T73, since the change in the size is only very small and might be mainly caused by a change in the invisible outer shell of the droplets. Therefore, it can be speculated by the following explanation: with dilution, the interaction radii increase due to redistribution of EtOH between the interface and the continuous aqueous phase according to equilibrium conditions, which is also expected to be less pronounced at decreased EtOH content (by moving from lines D64 and T64 to the lines D73 and T73). This loss of available co-surfactant in the interfacial area upon dilution would imply a 'true (slight) growth' of the droplets caused by an increase in aggregation number as observed in lines D64 (note: only the shrinking droplet core could be observed in the PDDFs in Fig. 3b) and T64. Because it was not feasible to detect such true growth in lines D73 and T73, we can consequently not exclude that the 
apparent growth has to be solely attributed to a decrease in the solvation layer.

C. Effect of oleic phase composition. The effect of oleic phase composition and concentration on the microstructure of two $\mathrm{O} / \mathrm{W}$ microemulsions was investigated next by changing the oleic phase $(R(+)$-limonene and ethanol) composition from a constant weight ratio of $1 / 1$ (see the dilution line T64 in Fig. 1) to $1 / 2$ (dilution line E64, more ethanol). Fig. 6a shows the scattering curves for both mixing ratios (T64 and E64) at 80 and 90 wt. \% of aqueous phase. Already from the raw scattering data, it can be seen that at constant aqueous phase content, there is a shift of the peak position to higher $q$-values with decreasing the mixing ratio of $R(+)$-limonene to EtOH (shrinking structures), and the intensity is lower. The PDDFs and the corresponding results of the structure factor for these two microemulsion systems are presented in Fig. $6 \mathrm{~b}$ and Table 1. Upon substituting $R(+)$-limonene partially by ethanol, the droplets become smaller (see PDDFs and the values of $\mathrm{R}_{\mathrm{HS}}$ ), and the effective volume fractions $\left(\phi_{\text {eff }}\right)$ are slightly lower (see Table 1). Those results mean that ethanol partitions between mostly the continuous aqueous phase and the interface. It should be noted that the PDDFs in Fig. $6 \mathrm{~b}$ go negative because of the fact that the droplets are inhomogeneous with respect to their electron density profile. This core shell structure has a negative electron density difference inside the core, and the shell only has a small electron density difference in the surrounding aqueous phase. The positive final part is often close to zero, when these particles are elliptical (slightly elongated). This can be proven by model calculations. This effect which is special for SAXS data also makes a precise determination of
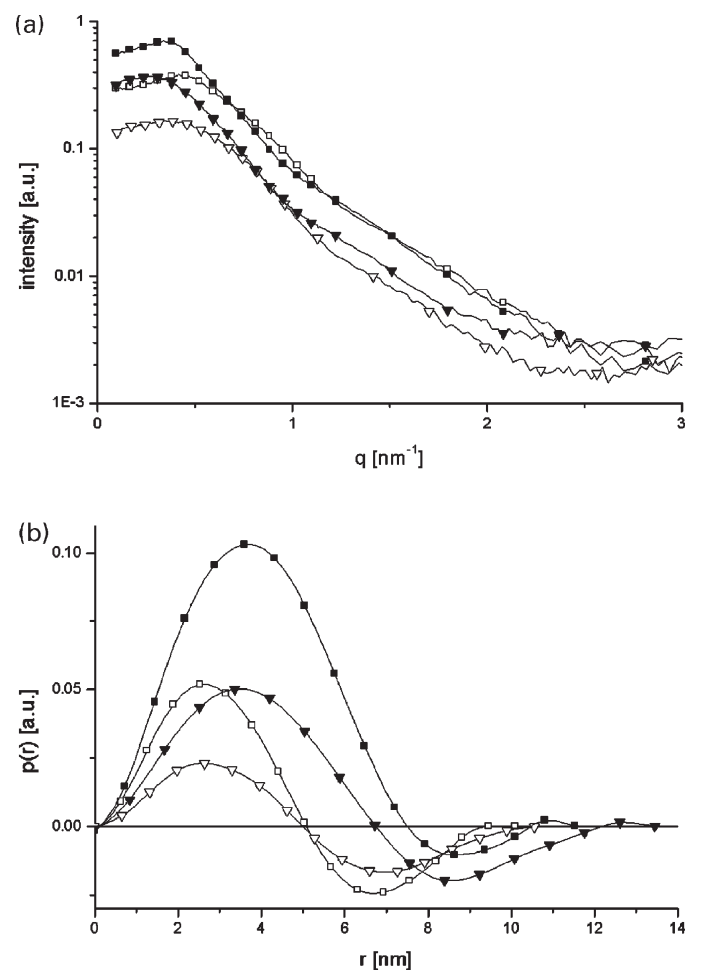

Fig. 6 Effect of oil phase composition in the microstructure of two $\mathrm{O} / \mathrm{W}$ microemulsions based on Tween 60 that contain $80(\boldsymbol{\square})$ and 90 ( $\boldsymbol{\nabla}$ ) wt. \% aqueous phase (water/PG at a constant weight ratio of 1 / 1 ) and an oleic phase composed of $R(+)$-limonene/ethanol at a weight ratio of $1 / 1$ (see dilution line T64), and $1 / 2$ (two $\mathrm{O} / \mathrm{W}$ microemulsions contain $80(\square)$ and $90(\nabla)$ wt.\% aqueous phase, this dilution line denoted as E64), respectively. (a) Experimental slit smeared scattering curves after subtraction of the background and solvent scattering for these samples, and (b) The pair distance distribution functions (PDDFs) for the corresponding systems.
$D_{\max }$ impossible. It will be very interesting in future to study these systems by SANS to gain more detailed information.

These results are in good agreement with our recent report on the effect of ethanol content on the microstructure of fivecomponent glycerol-based food-grade microemulsions. ${ }^{57}$ There, we found that ethanol redistributes mainly between the aqueous phase (water plus glycerol) and the interface-headgroup region of the surfactant. It was concluded that a considerable part of it is incorporated into the interfacial film, which leads to an increase in the available interface and results in a higher number of micelles with shrinking size. ${ }^{57}$ These results are also in good agreement with a previously reported study, ${ }^{33}$ which claims that short chain alcohols tend to penetrate into the palisade layer of the aggregates and the micelles are downsized. ${ }^{33}$

\section{Small angle neutron scattering (SANS)}

SANS is considered as a good tool in characterizing and extracting information from structuring processes of microemulsions and liquid crystals in recent years. ${ }^{44}$ We also used SANS in the present study, as neutrons offer an additional advantage over X-rays in being able to increase the contrast by using deuterated polar materials (EtOH, PG, and water) in our systems. The GIFT method was again applied to gain insight on the shape and size parameters from SANS data and to study how the presence of $\mathrm{PG}$, and $\mathrm{EtOH}$ influence the structure of aqueous micelles (or oil-swollen aqueous micelles) based on Tween 80 . The compositions of the investigated samples are listed in Table 2.

We started our SANS investigation with Tween 80-based aqueous micelles that contain $20 \mathrm{vol} \%$ Tween 80 (these are oil-free pseudo-binary systems). Fig. 7a shows the SANS data

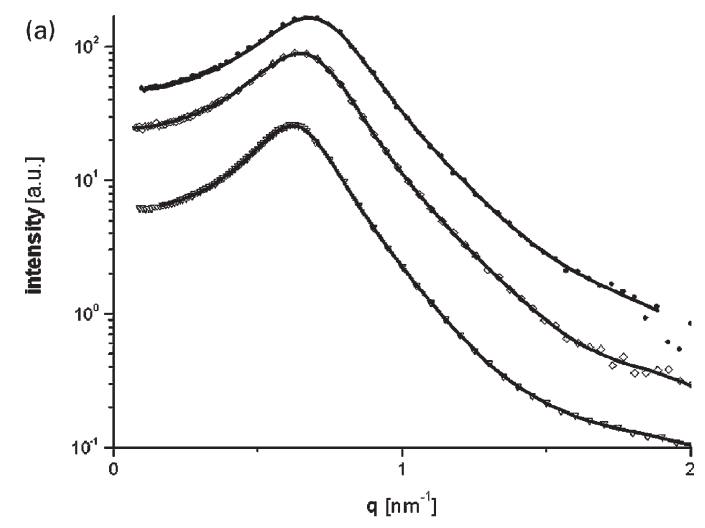

(b)

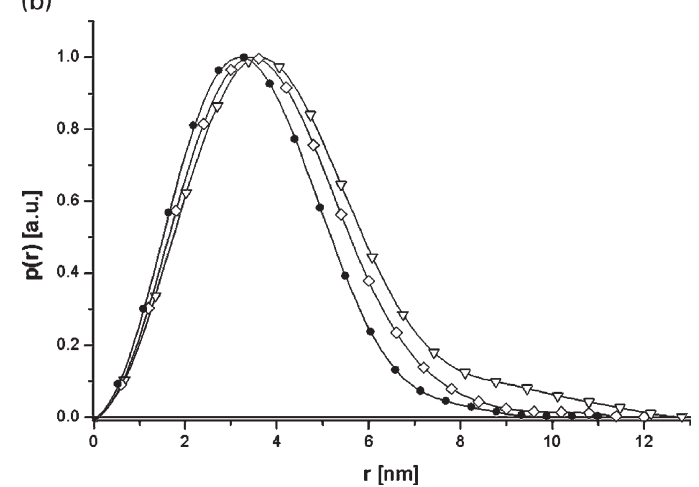

Fig. 7 (a) SANS data (symbols) together with fits from GIFT (solid lines) obtained from Tween 80 -based aqueous micelles with changing the aqueous phase composition (replacing water with PG), and (b) pair distance distribution functions normalized to the same height: effect of PG on the microstructure as measured by SANS of these aqueous micelles. The aqueous composition of these systems: $(\nabla)$ water, $(\diamond)$ water/PG (3/1); and $(\bullet)$ water/PG (1/1). 
(symbols) together with the fits from GIFT (solid lines). In these sample line, the structural evolution as a function of the aqueous phase composition (water plus PG) was studied (starting from pure $\mathrm{D}_{2} \mathrm{O}$ and consequently replacing water increasingly by $\mathrm{PG}$ up to a mixing ratio of $1 / 1$ ). Fig. $7 \mathrm{~b}$ shows the normalized PDDFs of these pseudo-binary systems: increasing the PG content leads to shrinkage of the micelles. It can be seen from this figure that $D_{\max }$ is reduced from 13 $\mathrm{nm}$ to $9.5 \mathrm{~nm}$ by replacing $\mathrm{D}_{2} \mathrm{O}$ by a mixture of $1 / 1 \mathrm{D}_{2} \mathrm{O} / \mathrm{d}-$ PG. In addition, the micelles transform from a prolate to a quite globular shape with increasing PG content in the aqueous phase (ratio of the position of the maximum in the PDDF to $D_{\max }$ is increased). It should be noted that asymmetry of the PDDFs, in principle, could either stem from elongation of the droplets, as well as from the presence of polydisperse spheres. In addition, the expected rather continuous change in the scattering length density profile at the particle surface, in contrast to a rapid change in the scattering length density from droplet to continuous phase (step function), can make the PDDF of homogenous spheres appear a little asymmetric. However, since the system investigated here by SANS can be almost regarded as micellar solution (it contains only a very small amount of oil), the major reason for asymmetry can be most likely attributed to an elongation of the droplets.

The shrinkage observed in the PDDF, can also be observed in the interaction parameters derived from the obtained structure factors (see Table 2 and Fig. 8): the interaction radius $\left(R_{\mathrm{HS}}\right)$ decreases from $4.8 \mathrm{~nm}$ to $4.2 \mathrm{~nm}$, and at the same time, the volume fraction of the dispersed phase $\left(\phi_{\text {eff }}\right)$ decreases to a lower extent. By combining these two parameters, it is possible to calculate the particle number density $\left(N_{\mathrm{p}} \mathrm{mm}^{-3}\right)$, which increases by about $30 \%$. Consequently, the presence of PG increases the number of micelles, and decreases their size and their total volume. The higher number of micelles at smaller size and consequently lower surfactant aggregation number can be explained in the following way: although most PG remains in the aqueous phase, a considerable part of PG is incorporated into the interfacial film; it has the function of a co-surfactant. ${ }^{34-40}$ It is more difficult to explain why this incorporation does not cause an increase in the effective volume fraction of the dispersed phase. For this, we have to keep some important aspects in mind: the effective volume fraction for the binary sample of $20 \mathrm{wt} . \%$ Tween 80 in water is $31 \mathrm{vol} \%$, which means that the micelles are highly hydrated (approximately one third is water!). Upon partially replacing water by $\mathrm{PG}$, many things change at the same time. As mentioned above, part of PG is incorporated into the interface; however, the main part stays in the aqueous phase, changing its polarity and interaction with the hydrophilic headgroups of the surfactant and, therefore, the nature of the solvation layer. The best explanation for a decreasing volume fraction is that the presence of PG in the system causes a reduction of the solvation layer by containing less water (dehydration).

The effect of ethanol on the microemulsion systems studied earlier by SANS ( $20 \mathrm{vol} \%$ Tween 80 and $1 \mathrm{vol} \% R(+)$-limonene in aqueous phase consisting of $\mathrm{D}_{2} \mathrm{O} / \mathrm{PG}$ at a constant ratio of $1 / 1$; composition is presented in Table 2) was investigated by replacing the aqueous phase partially by d-EtOH. The obtained SANS data (symbols) together with the fit from GIFT (solid lines) are shown in Fig. 9a, the corresponding PDDFs of these systems are given in Fig. 9b, and in addition, the respective structure factor parameters are given in Table 2 and illustrated in Fig. 8. In the sample without ethanol, the total volume of the droplets consists of $20 \mathrm{vol} \%$ Tween 80 and $1 \% R(+)$-limonene and the rest of the measured 29.5 $\mathrm{vol}^{\%} \%$ effective volume fraction is taken up by interfacial PG and the solvation layer of the droplets. Upon replacing aqueous phase (water plus PG) by ethanol, the particles shrink, change their shape from prolate to rather spherical and increase in their number (Fig. 8), which also leads to a
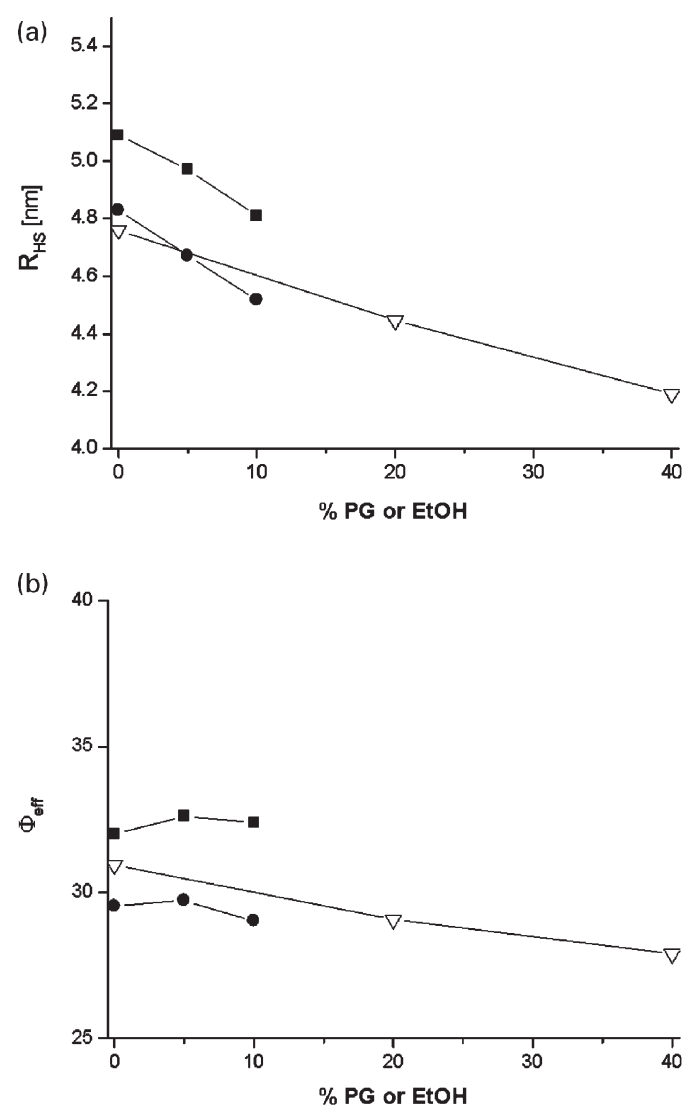

(c)

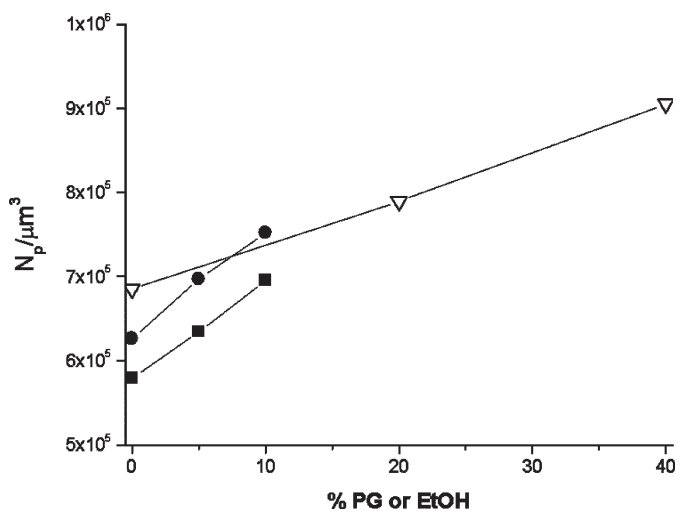

Fig. 8 Effect of d-EtOH and d-PG on the structure factor parameters of three different structured systems based on Tween 80 as were calculated from SANS data by GIFT: $(\nabla)$ impact of PG on pseudo-binary

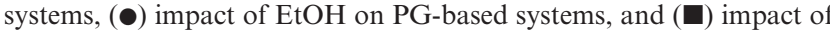
EtOH on PG-free systems. (a) the variation of the interaction radius $\mathrm{R}_{\mathrm{HS}}$, (b) the effective volume fraction $\phi_{\mathrm{eff}}$, and (c) the particle number density $\left(N_{\mathrm{p}} \mu \mathrm{m}^{-3}\right)$ with increasing d-EtOH or d-PG content.

reduction in the surfactant aggregation number (this can also observed in the decreased area in the PDDFs in Fig. 9b). For instance, increasing the amount of d-EtOH from 0 to 10 vol $\%$ reduces $D_{\max }$ from 13 to $8 \mathrm{~nm}$, respectively, and increases the ratio of the maximum in the PDDF to $D_{\max }$. These effects are very similar to those observed for PG, but unlike PG, added $\mathrm{EtOH}$ does not change the effective volume fraction of the dispersed phase (PG decreased it, most probably due to dehydration). We can directly conclude that most EtOH is dissolved the aqueous phase, while a part of it is incorporated into the interface, acting as co-surfactant. The similarity of the effects of $\mathrm{PG}$ and $\mathrm{EtOH}$, observed from the evaluation of SANS data, is confirmed and reinforced by the information obtained from their trends in the studied pseudo-ternary phase diagrams. ${ }^{25,26}$ It is very interesting to compare the results of 


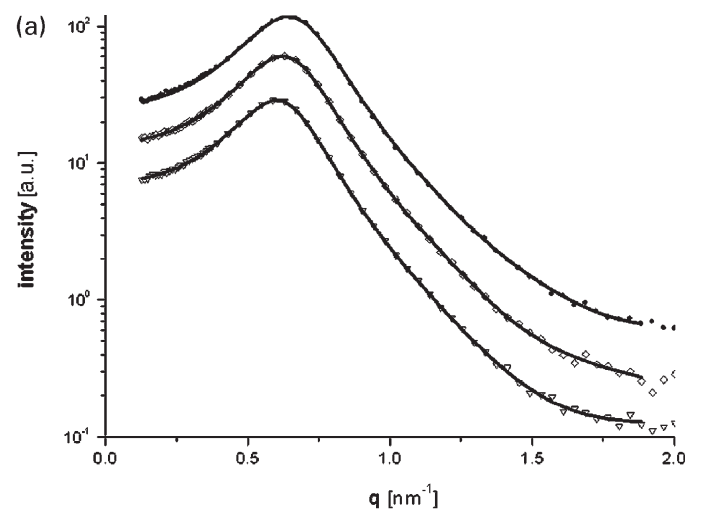

(b)

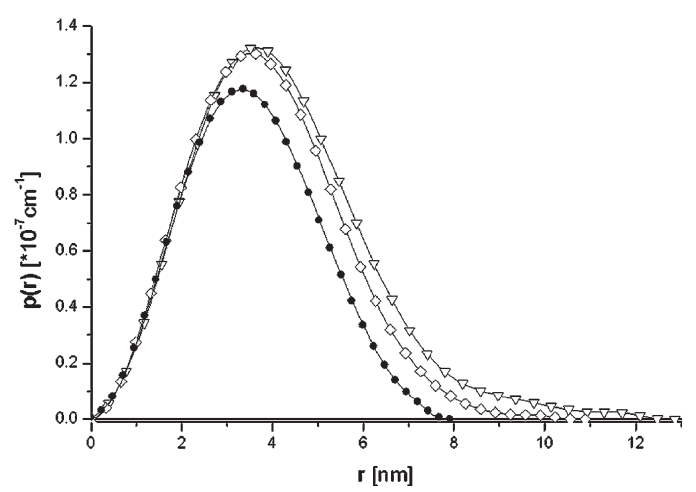

Fig. 9 (a) SANS data (symbols) together with fits from GIFT (solid lines) obtained from Tween 80-based aqueous micelles with replacing the aqueous phase (water plus PG) by ethanol, and (b)pair distance distribution functions: Effect of d-EtOH on the microstructure of Tween 80 -based microemulsions. EtOH content in these samples: $(\nabla) 0$ wt. $\%,(\diamond) 5$ wt. $\%$, and $(\bullet) 10$ wt. $\%$.

these samples to those in which the aqueous phase consisted of only $\mathrm{D}_{2} \mathrm{O}$ (PG-free systems, see Table 2). The absolute structure factor parameter values were found to be sensitive to the presence of PG (with more PG, both the effective volume fraction $\phi_{\text {eff }}$ and the interaction radii $R_{\mathrm{HS}}$ are decreased, the same trend that was observed in the system discussed above). However, increasing the d-EtOH content in these systems renders a very similar trend (Fig. 8), irrespective whether the aqueous phase contained d-PG or not. This leads us to the conclusion that the effects of EtOH and PG are rather independent, and when using a combination of these, their effects seem to simply add up in the concentration range studied.

\section{Conclusion}

The microstructure of Winsor IV O/W five-component microemulsions based on Tween 60 or Tween 80 (food-grade system), and Brij 96v (non-food grade system) has been studied by small angle scattering (SAXS and SANS) methods. We were able to extract information on the effect of short chain alcohol (ethanol, EtOH) and water-soluble polyol (polypropylene glycol, PG) on the size and shape as well as interaction parameters of different microemulsion systems.

Summarizing this work, we conclude the following:

\section{SAXS}

(i) Upon increasing the aqueous phase content of microemulsions along the various dilution lines that are presented in Fig. 1, there is an increase in the overall size of all microemulsion droplets (which can be attributed to a redistribution of ethanol). The microemulsion droplet size based on Tween 60 is bigger than that on Brij $96 \mathrm{v}$ at equal weight fraction. (ii) The surfactant concentration plays an important role: upon increasing the surfactant concentration (mixing ratio of surfactant to oleic phase at constant aqueous phase content), the effective volume fractions of dispersed phase stay constant, while the interaction radii decrease, leading to an increase in the number particle density. Therefore, the interfacial area is increased as expected.

(iii) Effect of ethanol (EtOH) in this system: it is highly soluble in the oil, but even better in the aqueous phase and we could prove that considerable parts also redistribute into the interface, i.e., ethanol is acting as a co-surfactant, depending on the ratio of the other components.

\section{SANS}

(i) Upon increasing the PG content in the aqueous phase, we observed that there is an increase in the micelles' number, a transformation from prolate to quite globular droplets and also shrinkage in the micellar size, leading to the conclusion that also part of PG is incorporated into the interface.

(ii) There is a strong similarity between EtOH and PG: while most of these two components remains in the aqueous phase, both are partially incorporated into the interface, rendering the droplets' shape more globular and their size smaller. The only observed difference between these two water-soluble alcohols was that a substitution of aqueous phase by EtOH does not lead to a significant change in the effective volume fraction, while it is decreased by PG because PG lowers the hydration level in the solvation layer (dehydration).

(iii) EtOH and PG influence the microstructure of our investigated samples independently.

\section{Acknowledgements}

This research was supported by a grant from the Ministry of Science, Israel, the Austrian Science Fund FWF (M711NO3) and was also supported by the Nestlé Research Center, Lausanne, Switzerland. We acknowledge the PSI for providing the facilities used in the neutron experiments and special thanks go to our local contacts Thomas Keller and Vinod Aswal.

\section{References}

1 K. Shinoda and B. Lindman, Langmuir, 1987, 3, 135-149.

2 C. Solans, R. Pons and H. Kunieda, in Industrial Applications of Microemulsions, ed. C. Solans and H. Kunieda, Marcel Dekker, New York, 1997, vol. 66, pp. 1-17.

3 S. Ezrahi, A. Aserin and N. Garti, in Handbook of Microemulsion Science and Technology, ed. P. Kumar and K. L. Mittal, Marcel Dekker, New York, 1999, pp. 185-246.

4 O. Regev, S. Ezrahi, A. Aserin, N. Garti, E. Wachtel, E. W. Kaler, A. Khan and Y. Talmon, Langmuir, 1996, 12, 668-674.

5 M. Kahlweit, G. Busse, B. Faulhaber and J. Jen, J. Phys. Chem., 1996, 100, 14991-14994.

6 J. F. Billman and E. W. Kaler, Langmuir, 1991, 7, 1609-1617.

7 S. R. Dungan, in Industrial Applications of Microemulsions, ed. C. Solans and H. Kunieda, Marcel Dekker, New York, 1997, vol. 66 , pp. $148-170$.

8 M. R. Gasco, in Industrial Applications of Microemulsions, ed C. Solans and H. Kunieda, Marcel Dekker, New York, 1997, vol. 66 , pp. 97-122.

9 J. Alander and T. Warnheim, J. Am. Oil Chem. Soc., 1989, 66 1656-1660.

10 J. Alander and T. Warnheim, J. Am. Oil Chem. Soc., 1989, 66, $1661-1665$.

11 C. Malcolmson and M. J. Lawrence, Colloids Surf. B, 1995, 4, 97-109.

12 W. Warisnoicharoen, A. B. Lansley and M. J. Lawrence, Int. J. Pharm., 2000, 198, 7-27.

13 C. von Corswant, S. Engström and O. Söderman, Langmuir, 1997, 13, 5061-5070. 
14 C. von Corswant and O. Söderman, Langmuir, 1998, 14, 3506-3511.

15 S. Hamdan, R. Lizana and C. R. Laili, J. Am. Oil Chem. Soc., 1995, 72, 151-155.

16 N. Kanei, Y. Tamura and H. Kunieda, J. Colloid Interface Sci., 1999, 218, 13-22

17 Y. Tokuoka, H. Uchiyama, M. Abe and S. D. Christian, Langmuir, 1995, 11, 725-729.

18 R. F. Joubran, D. G. Cornell and N. Parris, Colloids Surf. A, 1993, 80, 153-160.

19 S. F. Trevino, R. Joubran, N. Parris and N. F. Berk, J. Phys. Chem. B, 1998, 102, 953-960.

20 P. P. Constantinides and J. P. Scalart, Int. J. Pharm., 1997, 158, 57-68.

21 S. Prichanont, D. J. Leak and D. C. Stuckey, Colloids Surf. A, 2000, 166, 177-186.

22 K. M. Park and C. K. Kim, Int. J. Pharm., 1999, 181, 173-179.

23 A. Radomska and R. Dobrucki, Int. J. Pharm., 2000, 196, $131-134$.

24 M. Trotta, S. Morel and M. R. Gasco, Pharmazie, 1997, 52 , 50-53.

25 N. Garti, A. Yaghmur, M. E. Leser, V. Clement and H. J. Watzke, J. Agric. Food Chem., 2001, 49, 2552-2562.

26 A. Yaghmur, A. Aserin and N. Garti, Colloids Surf. A, 2002, 209, 71-81.

27 A. Yaghmur, A. Aserin and N. Garti, J. Agric. Food Chem., 2002, 50, 2878-2883.

28 A. Spernath, A. Yaghmur, A. Aserin, R. E. Hoffman and N. Garti, J. Agric. Food Chem., 2002, 50, 6917-6922.

29 A. Spernath, A. Yaghmur, A. Aserin, R. E. Hoffman and N. Garti, J. Agric. Food Chem., 2003, 51, 2359-2364.

30 A. Yaghmur, A. Aserin, I. Tiunova and N. Garti, J. Therm. Anal. Cal., 2002, 69, 163-177.

31 A. Yaghmur, A. Aserin, B. Antalek and N. Garti, Langmuir, 2003, 19, 1063-1068.

32 T. Iwanaga, M. Suzuki and H. Kunieda, Langmuir, 1998, 14, 5775-5781.

33 K. Aramaki, U. Olsson, Y. Yamaguchi and H. Kunieda, Langmuir, 1999, 15, 6226-6232.

34 A. Martino and E. W. Kaler, J. Phys. Chem., 1990, 94, 1627-1631.
35 A. Martino and E. W. Kaler, Langmuir, 1995, 11, 779-784

36 R. Strey, Colloid Polym. Sci., 1994, 272, 1005-1019.

37 R. Ivanova, B. Lindman and P. Alexandridis, Langmuir, 2000, 16 3660-3675.

38 P. Alexandridis, R. Ivanova and B. Lindman, Langmuir, 2000, 16, 3676-3689.

39 R. Nagarajan and C.-C. Wang, J. Colloid Interface Sci., 1996 , 178, 471-482.

40 R. Nagarajan and C.-C. Wang, Langmuir, 2000, 16, 5242-5251.

41 A. Ray and G. Nèmethy, J. Phys. Chem., 1971, 75, 809-815.

42 A. Martino and E. W. Kaler, Colloids Surf. A, 1995, 99, 91-99.

43 C. Mathew, Z. Saidi, J. Peyrelasse and C. Boned, Phys. Rev. A, 1991, 43, 873-882.

44 T. Hellweg, Curr. Opin. Colloid Interface Sci., 2002, 7, 50-56.

45 A. Bergmann, G. Fritz and O. Glatter, J. App. Cryst., 2000, 33, 1212-1216

46 G. Fritz, A. Bergmann and O. Glatter, J. Chem.. Phys., 2000, 113, 9733-9740.

47 O. Glatter, D. Orthaber, A. Stradner, G. Scherf, M. Fanun, N. Garti, V. Clement and M. E. Leser, J. Colloid Interface Sci., 2001, 241, 215-225.

48 J. Brunner-Popela, R. Mittelbach, R. Strey, K.-V. Schubert, E. W. Kaler and O. Glatter, J. Chem. Phys., 1999, 110, 10623-10 632.

49 A. Bergmann, D. Orthaber, G. Scherf and O. Glatter, J. Appl. Crystallogr., 2000, 33, 869-875.

50 J. Brunner-Popela and O. Glatter, J. Appl. Crystallogr., 1997, 30, $431-442$.

51 O. Glatter, J. Appl. Crystallogr., 1979, 12, 166-175.

52 O. Glatter, in Small Angle X-ray Scattering, ed. O. Glatter and O. Kratky, Academic Press, London, 1982, p. 119-196.

53 E.W. Kaler, in Modern Aspects of Small-Angle Scattering, ed. H. Brumberger, KluwerPress, Dordrecht, 1994.

54 B. Weyerich, J. Brunner-Popela and O. Glatter, J. App. Cryst., 1999, 32, 197-209.

55 O. Glatter, G. Fritz, H. Lindner, J. Brunner-Popela, R. Mittelbach, R. Strey and S. Egelhaaf, Langmuir, 2000, 16, 8692-8701.

56 H. Lindner, G. Fritz and O. Glatter, J. Colloid Interface Sci., 2001, 242, 239-246.

57 L. de Campo, A. Yaghmur, N. Garti, M. E. Leser, B. Folmer and O. Glatter, J. Colloid Interface Sci., in press. 